\title{
Research on Reliability of Air Control Communication System Based on Civil Aviation
}

\author{
Xiaochi Feng \\ Civil Aviation Shanxi Air Traffic Control Branch, Taiyuan 030031, Shanxi, China
}

\begin{abstract}
In the civil aviation industry of our country, the air traffic control communication system is an important information transmission mode of ground-to-air communication and plane communication. In order to ensure the safe and reliable operation of the communication system, this paper mainly analyzes the safety impact of the civil aviation air control communication system, and gives feasible treatment measures to effectively improve the communication mode, and reduce the impact on various influencing factors.
\end{abstract}

Keywords: Civil aviation, Air control, Communication system, Reliability.

\section{Introduction}

According to the function of the Civil Air traffic control communication system, its aim is to establish the communication link of Resistance Communication and plane communication to guarantee the stable transmission of signals, thus providing the stable guarantee for the air traffic control service, but in order to improve the reliability and authenticity of the signal transmission, we must pay attention to the management and maintenance of the communication system, avoid the influence of various interference factors on the communication system, and ensure the normal communication.

\section{Influactors Influencing Reliability of Civil Aviation Air Control Communication System}

\subsection{Intermodulation Interference}

The common reason in civil air control communication system is mutual coordination interference, which is mainly due to the circuit system, which is mainly divided into receiver coordination interference and transmitter coordination interference, coupling reaction through system setting, which causes or affects the normal acquisition efficiency of information, which also has a great impact on the safety of civil air control communication system. This paper analyzes the transmitter intermodulation interference. In the normal operation of the transmitter device, various equipment information resources and transmitter channels interact, which makes the equipment information influence each other, and increases the difficulty of collecting information resources, thus directly affects the operation stability of civil aviation air control communication system. After practical application, it can be seen that the direct coupling reaction of signal and channel mainly affects the signal of the high frequency band. However, affected by the intermodulation interference, the civil aviation aircraft and he will have communication blocked, which has a serious impact on the civil aviation system, and even cause major safety risks.

\subsection{Beat Interference}

Traffic adjustment interference in the operation of civil aviation air control communication system is also the main factor affecting its stability. Therefore, people must pay attention to this influencing factor, and do a good job in the management of traffic adjustment interference factors. Intermodulation interference distorts the signal resource acquisition because the signal is transmitted to the terminal through the mixer. The intermediate frequency signal is difficult to filter the modulated signal. Therefore, this medium frequency signal will have a serious impact on the civil aviation air control communication system, leading to stability damage, and interference with the normal communication between the aircraft and him. Usually, the interference mainly appears in the input of the communication system, so, to effectively deal with the interference problem, we must pay attention to the control of the input, do a good job in data management and maintenance.

\subsection{Wave-channel Interference}

At present, the research and analysis method of secondary wave channel interference in civil aviation air control communication system is generally deeply studied, and the main reason for the interference problem is the medium and high frequency signal interference produced by interference and local vibration mixing, and the interference source is mostly produced by

\section{Reliability Study of Civil Aviation Air Control Communication System}

\subsection{Off-site Backup and Select the Operator}

For the operation stability of high frequency communication, first of all to build high frequency stations in different areas, so as to ensure different regional communication content, but to pay special attention to the establishment of the high frequency communication platform, each sector construction must maintain a good coverage area, so as to realize the comprehensive coverage of service function, but also help to improve the reliability of civil aviation air control communication system. In addition, we should also pay attention to the scientific selection of operators, which can also effectively improve the continuity and reliability of signal resources, and avoid the occurrence of system failures. Therefore, when choosing operators in the civil aviation system, it must choose the operation system with strong anti-interference ability, and technical technicians can 
effectively realize the system management through the dual trunk mode. The application of the dual trunk line mode can effectively guarantee the stable operation of the air communication system, can play an important role in the choice of operators and data backup, and can also meet the effective backup of multiple frequency bands, so as to improve the system stability.

\subsection{Equipment Dispersion and Signal Transmission}

In the operation of civil aviation air control communication system, the factors affecting its stability include the choice of equipment connection, can improve the interference ability of civil aviation air control communication system in parallel, therefore, to comprehensively improve the parallel effect of high frequency signals, can backup different modules in connection, ensure equipment connection, set multiple backup points in different areas of the sector, and adopt various connection methods to ensure the effective connection of communication equipment, so as to achieve efficient signal acquisition.

In addition, the reduction of the signal transmission link will also cause certain interference to the civil aviation air control communication system. Therefore, the signal transmission process should also be paid attention to reducing the transmission link, so as to effectively improve the signal transmission efficiency and enhance the stability of the system. It has been proved through practice that the more cumbersome the signal transmission link, the worse the transmission reliability. Therefore, the civil aviation air control communication system must pay attention to the scientific design of the signal transmission link, simplify the transmission process of each frequency band, reduce the signal jump transmission phenomenon, and improve the stability of the system.

\subsection{Strengthen the Reliability of the Dialogue System}

Based on the high-frequency signal transmission of China's civil aviation air control communication system, it will also be disturbed by all kinds of signals, which leads to frequent signal detection problems in reaching the dispatching platform, so it also affects the civil aviation air traffic control system, resulting in insufficient scheduling from time to time. To this end, the relevant personnel must face up to the improvement of the dialogue system, and comprehensively improve the stability of the civil aviation air control communication system. Most of the current civil aviation air control communication system is high frequency signal, and the signal in transmission link loss, therefore, must actively build high stable communication system, such as through high frequency communication system setting, including internal assimilation and central transmission functions, improve the reliability of system reliability. In order to further improve the stability of the civil aviation air control communication system, a dialogue system can also be set up internally to conduct equipment maintenance in parallel, so as to ensure that the dialogue function of the civil

\section{Conclusion}

To sum up, with the comprehensive development of civil aviation in China, it brings people a good life experience, and the civil aviation air control communication system is a bridge to ensure the good connection between civil aviation aircraft and towers. In order to effectively improve the reliability of civil aviation air control communication system, attention must be paid to analyzing the main factors affecting the reliability of the system, and take effective countermeasures to strengthen the operation reliability of civil aviation air control communication system and promote the comprehensive development of civil aviation.

\section{References}

[1] Yao Yunfei, Yang Wei, Chen Liqiang. Design and performance analysis of adaptive anti-jamming system in China [J]. Telecommunications technology, 2019, 59(12): 1385-1390.

[2] Chen Fei. Explore the development of civil aviation ground and air communication technology in the new period [J]. Engineering Construction and Design, 2019, (22): $259-260$. 